\title{
Toward an AI-Enabled SDN-based 5G \& IoT Network
}

\author{
Bao-Shuh Paul Lin ${ }^{1}$ \\ ${ }^{1}$ Department of Computer Science, National Chiao Tung University, Hsinchu, Taiwan \\ Correspondence: Bao-Shuh Paul Lin, Department of Computer Science, National Chiao Tung University, Hsinchu, \\ Taiwan. Tel: 886-3-573-1929.
}

Received: November 19, 2020 Accepted: November 30, 2020 Online Published: December 10, 2020

doi:10.5539/nct.v5n2p7 URL: https://doi.org/10.5539/nct.v5n2p7

\begin{abstract}
Artificial Intelligent technology has been applied to a wide variety of applications including products, systems, and services of the Information \& Communications Technology (ICT) and non-ICT or traditional industries. The benefits of these applications includes performance improvement, optimization, intelligent.The 5G mobile/wireless networks have intelligent features of network slicing and edge computing because of network equipment system vendors apply AI technology to the mobile systems. On the other hand, many traditional industries have benefits from AI technology in particular Machine Learning (ML) and Deep Learning (DL). Recently in the agriculture, healthcare, finance, and many other applications and services have adopted $\mathrm{AI} / \mathrm{ML} / \mathrm{DL}$ technology even with the integration of $5 \mathrm{G}$ and Internet of Things (IoT). This article focuses on the system architecture and design of open networking $(\mathrm{ON})$ solution of 5G, the approach of SDN/NFV-based 5G and IoT and how $\mathrm{AI} / \mathrm{ML}$ interact with $5 \mathrm{G} / \mathrm{IoT}$ and learns from these. We call this interaction as SDN-based 5G/IoT Network AI or AI-enabled SDN-based 5G/IoT Network.

Keywords: Artificial Intelligence (AI), Machine Learning (ML), Deep Learning (DL), the Fifth Generation Mobile System (5G), Internet of Things (IoT), Software Defined Network (SDN), Network Function Virtualization (NFV)

\section{Introduction}

Artificial intelligent (AI) is a great technology and applications for which existing solutions require better automation or optimization and there is no good solution by using traditional approaches. So, when people say AI in Network really means using AI techniques to assist network operations, configuration, and management or to play a supporting role. On the other hand, Network with machine learning (ML), deep learning (DL) and other AI techniques used to manage network to be predictive and proactive, then AI/ML/DL becomes the driving force of network operations and management or the enabler of network operations. We call this kind of network is an AI-enabled Network (Yao et al., 2018; Zheng et al., n.d.). AI is also an interdisciplinary science with multiple approaches but the advancements in ML and DL are creating a paradigm shift virtually every sector of the technology industry.
\end{abstract}

\section{Networking Trends \& Directions}

\subsection{Open Network and $5 G$}

It has been a paradigm shift in the network industry, from a proprietary networking with vendor-dependent soft-ware/hardware to vendor-independent open source and white box switches as illustrated in Figure 1. The white box is the commodity hardware that is available from network equipment ODM vendors. On the other hand, the open source software executed on the commodity hardware in the control plane (with controller) of SDN can be very complex. Currently, there are three (3) well-developed SDN controller operating systems (OS) open source available including, ONOS, ODL, and RYU developed by various open source foundations.

The development direction from proprietary networking to open source based networking called Open Networking (ON). The first ON solution for 5G (in Japan) was proposed by Rakuten Mobile in September, 2019 and granted an operator license. Right now Rakuten is busy in the deployment of base stations and expect to provide the full coverage of Japan for mobile services in 2021. 

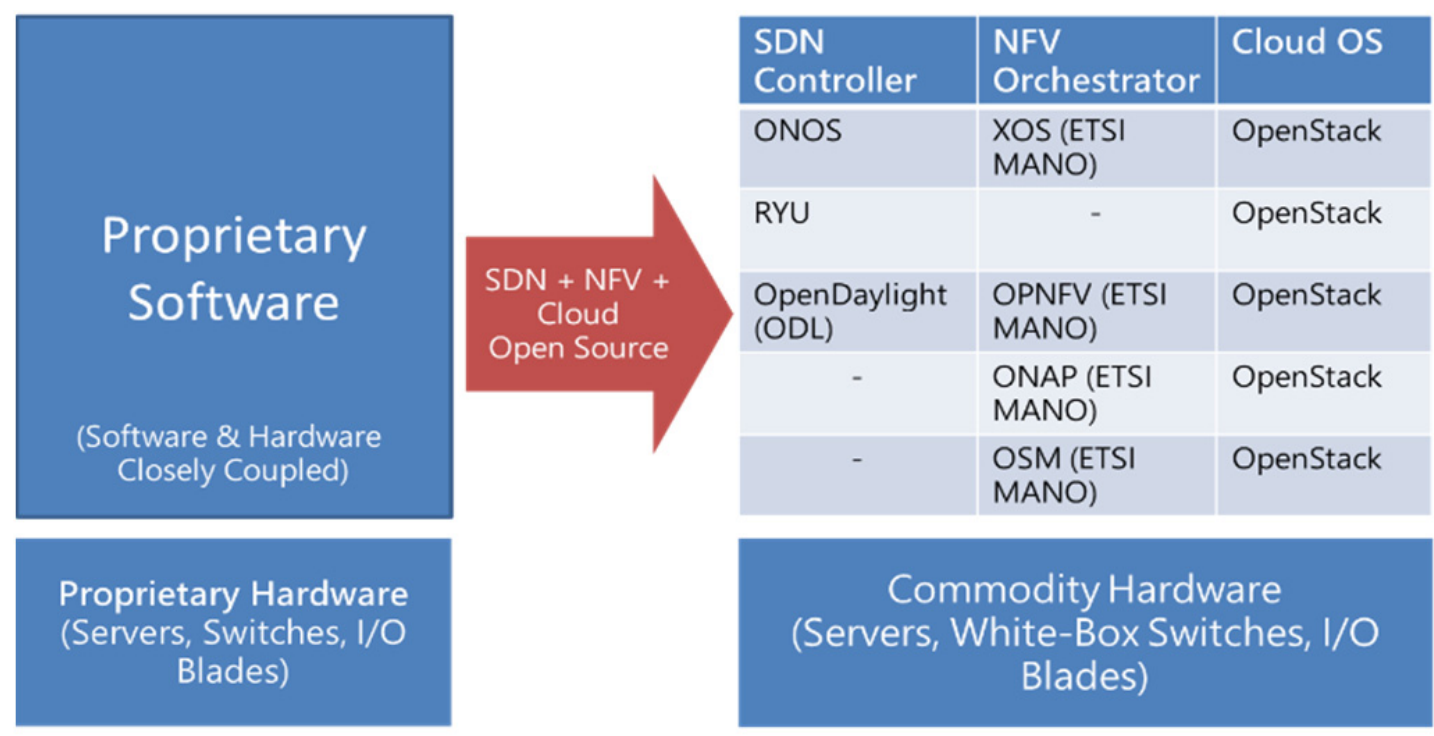

Figure 1. Open Networking (ON) Efforts

\subsection{Interne of Things (IoT)}

Figure 2 shows the definition of Internet of Things (IoT) and some examples Until now, most of the systems of Internet of Things (IoT) applications snd services are relying on $4 \mathrm{G} / \mathrm{LTE}$ or even $3 \mathrm{G}$ as the gateway to transport data to the destination for computing and applications creation and execution. Figure 3 illustrates wireless sensor network (WSN) which collects data and/or signals from devices and use 4G/LTE and 5G as the gateway to network and services platform for further application creation. Its equivalent 4 Layer Model of IoT is shown in Figure 4 (Akyildiz, 2017). Those 4 layers are layer 1: sensing and identification, layer 2: network construction, layer 3 : information processing and layer 4: integrated application.

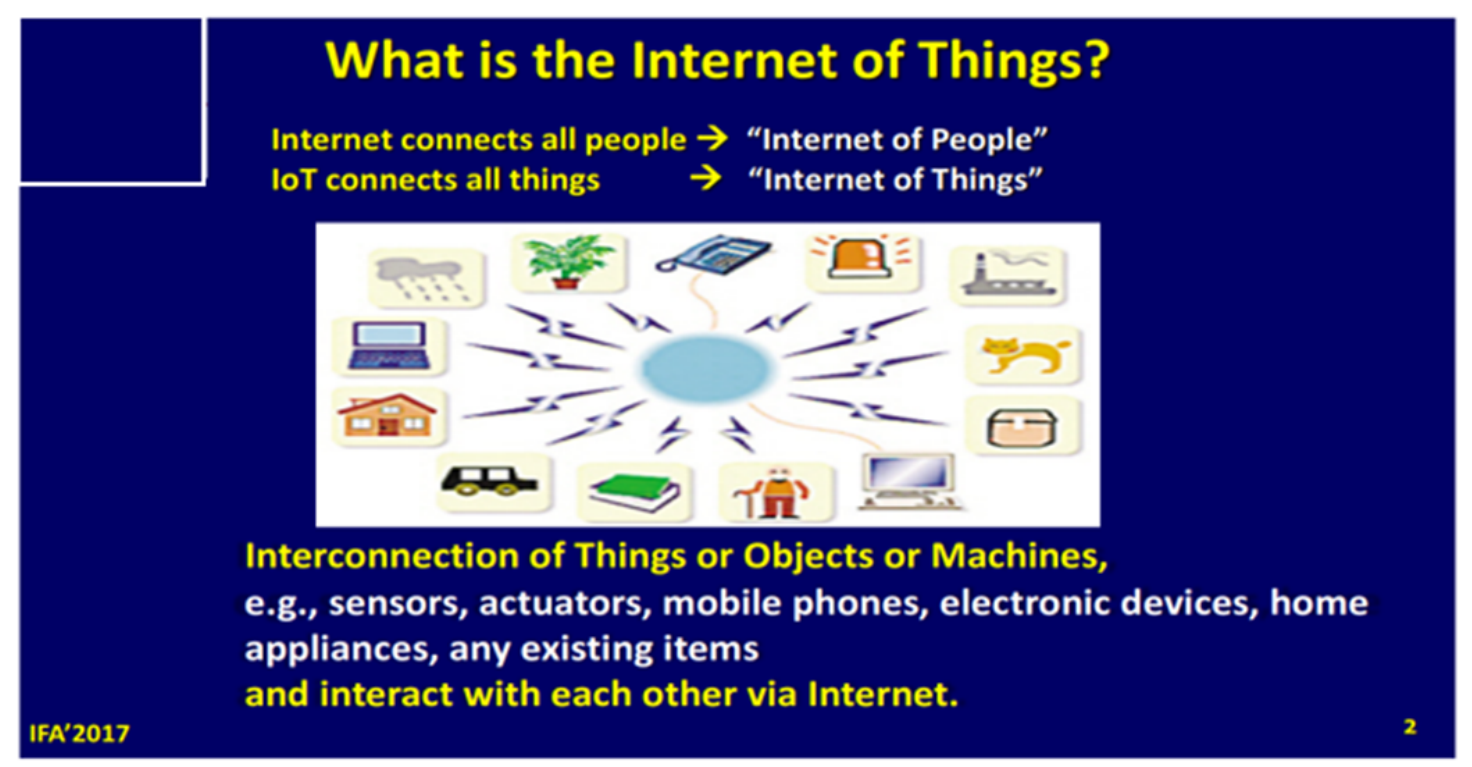

Figure 2. Internet vs. Internet of Things (Akyildiz, 2017) 


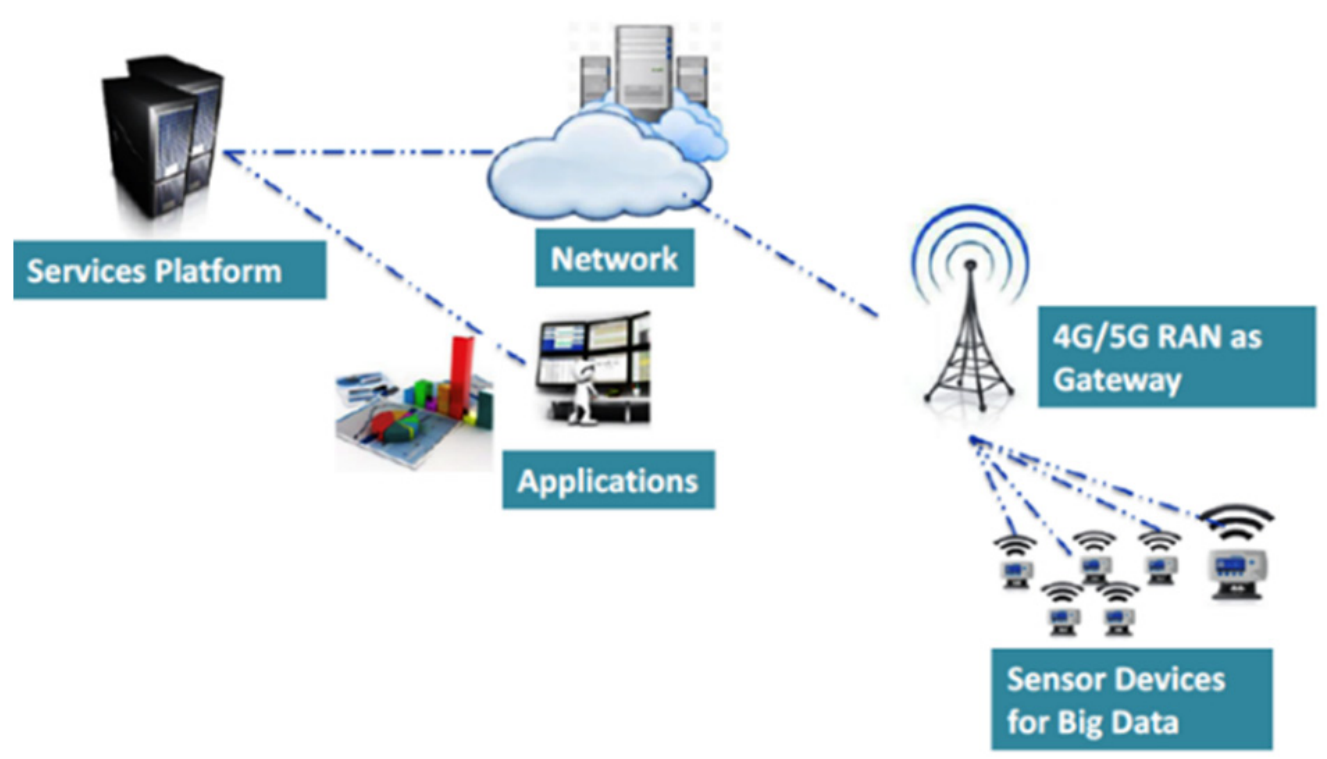

Figure 3. IoT Conceptual and Reference Architecture

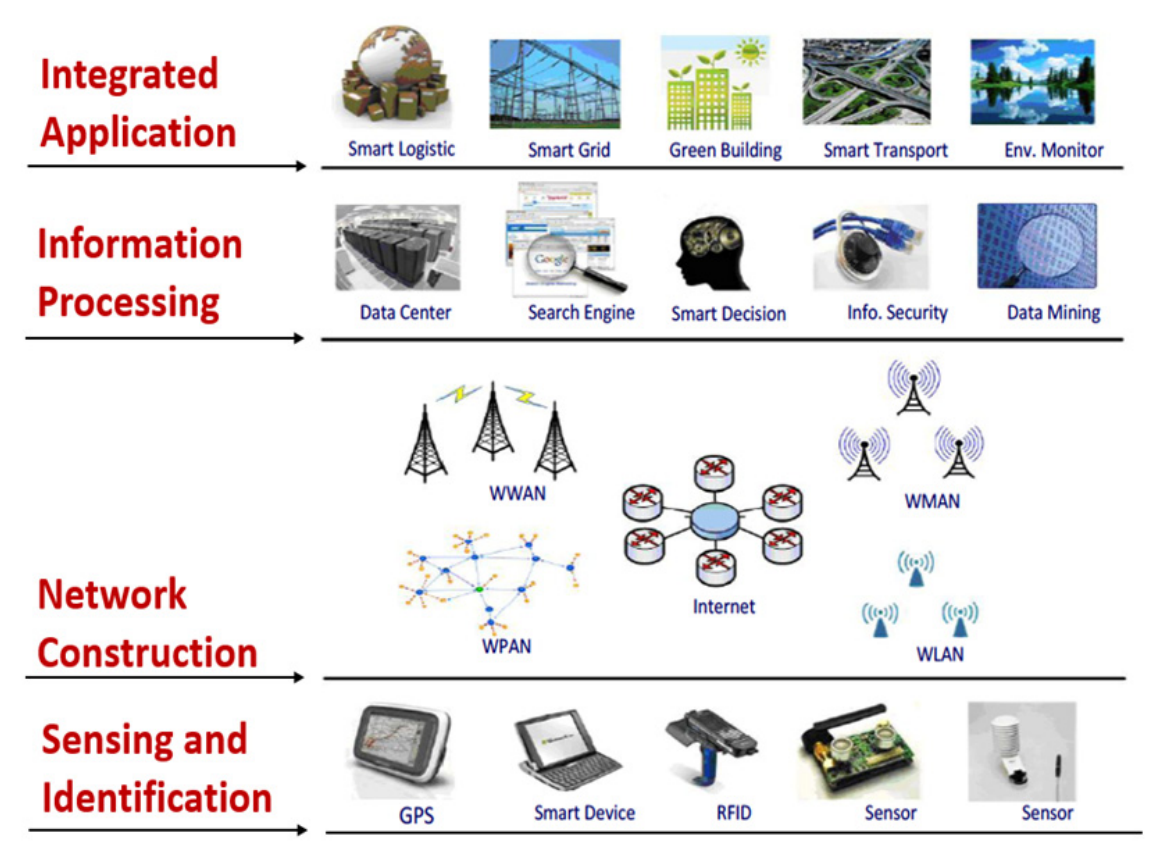

Figure 4. 4-layer model of IoT (Akyildiz, 2017)

\subsection{SDN-Based $5 G$}

In this article SDN-Based $5 \mathrm{G}$ means $5 \mathrm{G}$ network is comstructed and deployed by using open source code related to SDN/NFV to do the softwarization \& virtualization of $5 \mathrm{G}$. The network architecture of $5 \mathrm{G}$ consists of ORAN and virtual EPC (core network). ORAN is an open source radio acess network subsystem which separates RRH/RRU (remote radio head/remote radio unit) from BBU (baseband unit) and its core network is an available open source EPC. Figure 5 illustrates the open network architecture.of 5G. This open $5 \mathrm{G}$ network architectre creates the opportunity to be multi-vender to avoid lock-into one vender with proprietary solution.Also the separation of ORAN and EPC may create the new eco system of 5G industry. 


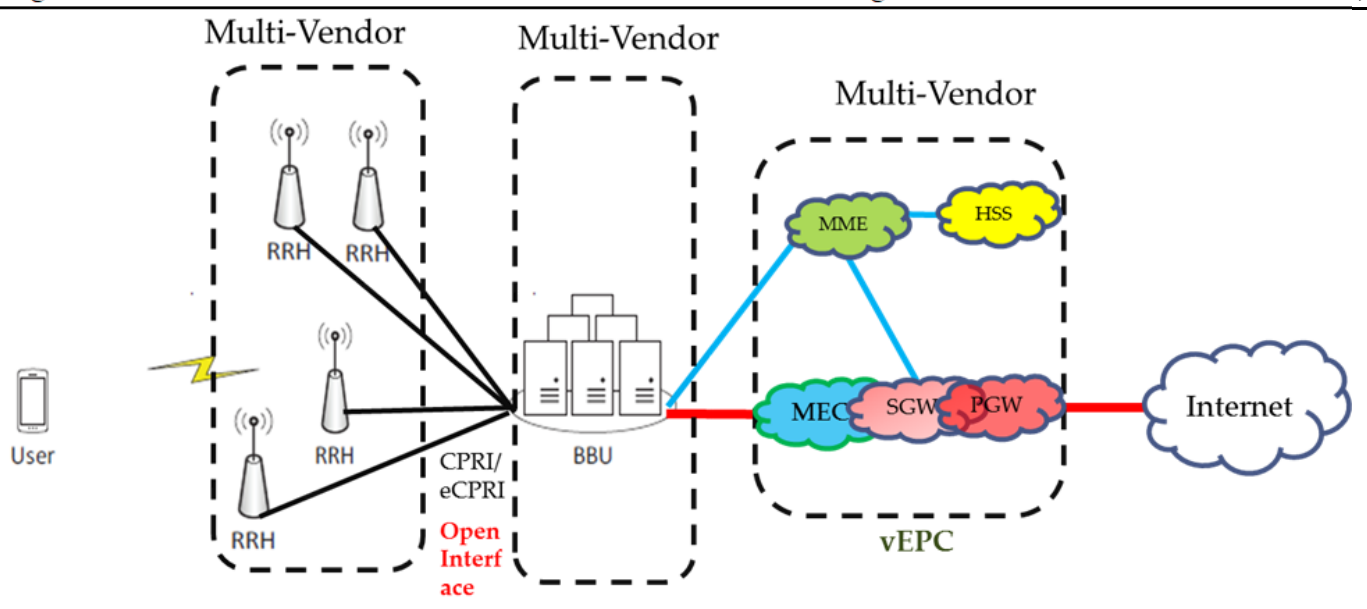

Figure 5. Open 5G network architecture

\section{Relationships among Al, ML Neural Networks and DL}

This section discusses the definitions of AI, ML, DL and their relationships and differences. AI is algorithms, programs, coding can make machine, objects and things more intelligence through learning and/or training. ML is a subset of AI while DL is a subset of AI and ML.

In practical AI system, these technologies are often supplemented by traditional software coding techniques to manage the AI system, to preprocess data used to drive the AI system, and to implement output a to adapters to effectively use the decision or recommendation output. Many forms of AI incorporate ML to enable the AI systems to better adapter to a complex potentially dynamic environment ML involves training or data acquisition that can modify machine behavior and comes many forms: supervised learning, unsupervised learning, reinforcement learning, and online learning.

Neural networking is a specific class of AI ML systems neural networks have shown promise in addressing complex data relationships. DL generally applies to large neural network with thousands of hidden layers wherein training occurs on each layer within the hidden nodes of a neural network. In the recent years deep learning neural networks have become the most promising approach to AI.

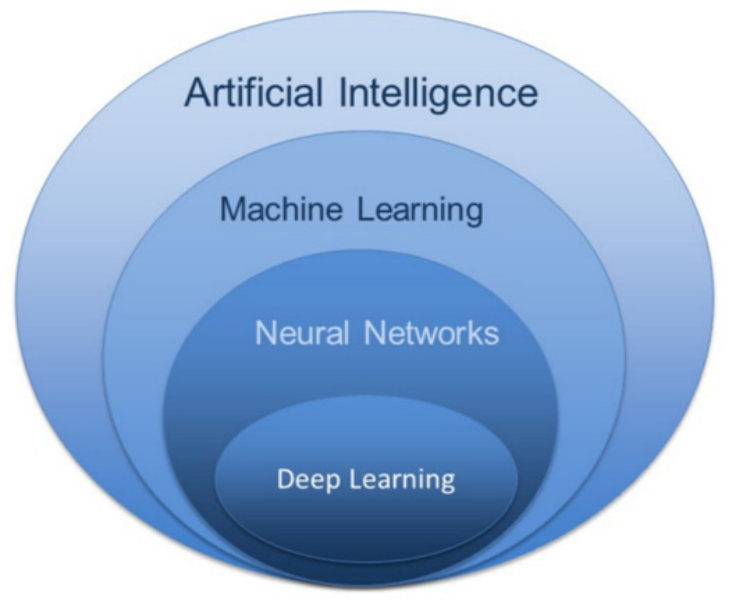

Figure 6. Relationships among Al, ML Neural Networks and DL (ATIS, 2018)

\section{AI in Network vs AI-enabled Network}

There are two ways to migrate AI software to the existed system. One way is try to use AI algorithms or techniques to assist the system to become more automation, more optimization, and better service. So, AI in network means what $\mathrm{AI}$ can assist network to complete network functions. The other way is using $\mathrm{AI} / \mathrm{ML} / \mathrm{DL}$ techniques to complete the network migration. So, AI-enabled Network means AI driven network operation and management. The characteristics of 3 approach to architect and design the network include: Traditional Networking without AI, 
AI in Network, AI-enabled Network are in the following:

1) Traditional Networking without AI/ML/DL: reactive, no intelligence, no optimization, error prone, inefficient

2) AI in Network is: semi-active, partial intelligent, local optimization, semi-automated

3) AI-enabled Network is: proactive, intelligent, global optimization, automated, scalable

Table 1 shows the comparison of 3 approaches.

Table 1. Comparison of Traditional Networking, AI in Network, and AI-enabled Network

\begin{tabular}{lccc}
\hline With AI & Traditional & AI in Network & AI-enabled Network \\
Characteristics & Networking & With AI/ML/DL & With AI/ML/DL \\
\hline Approach & without AI/ML/DL & semi-full active & proactive \\
Active & Reactive & semi-full intelligence & intelligence \\
Intelligence & no intelligence & local optimization & global optimization \\
Optimization & no optimization & semi-automated & automated \\
Automated & no automated & no-specific & scalable \\
Others & error prone inefficiency & &
\end{tabular}

\section{AI-enabled SDN/NFV 5 G and IoT Network}

The status of open source for 5G network architecture (Lin, 2019; Lin et al., 2019; 5G Americas whitepaper, 2019; B. S. P. Lin, F. J. Lin, \& Tung, 2016) is based on software defined network (SDN) for softwarization and network function virtualization (NFV) for virtualization principles. Together, these provide agility and flexibility in terms of resource placement and utilization. Figure 7 illustrates the simplified 5G network system architecture-based IoT applications. SDN-based 5G/IoT AI-enabled is shown in Figure 8.

5G/IoT AI-enabled can be used to manage 5G networks (Nelson, 2020) and IoT applications.

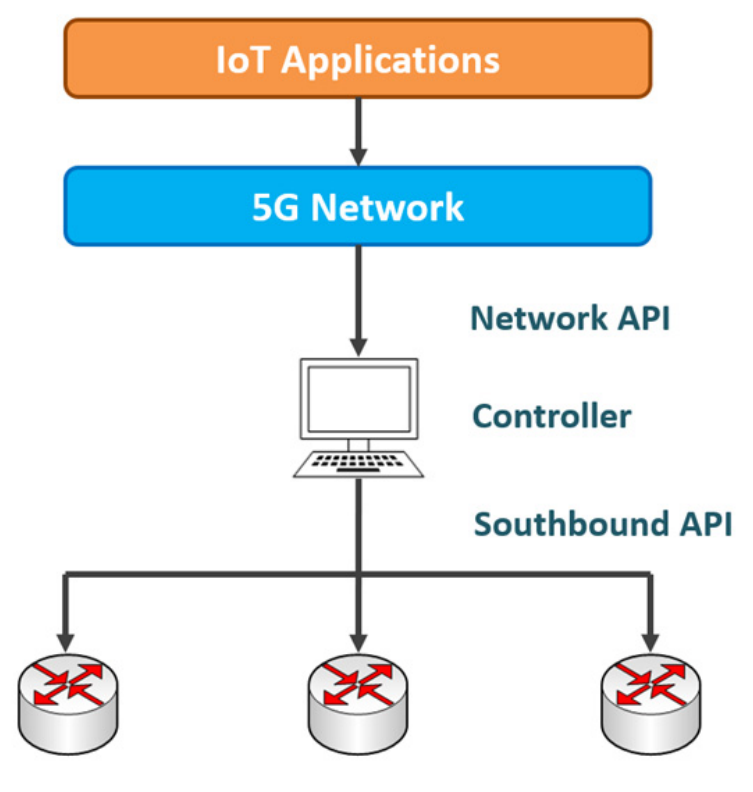

Network Switches

Figure 7. Simplified 5G SDN Architecture-based IoT Applications 


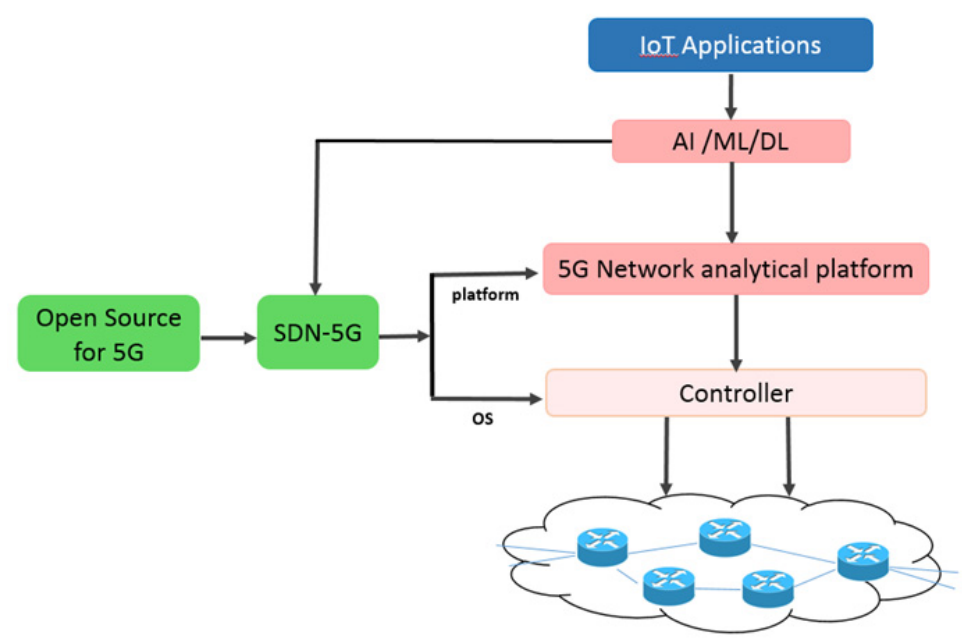

Figure 8. AI-enabled SDN-based 5G/IoT (Yao et al., 2018)

For example, network slicing different is a way to use different services on the same network infrastructure - voice and IoT. It's a kind of flexibility feature let different applications use varying bandwidth. Different latency maintain varying levels of reliability depending on need.

\section{Global AI Industry}

The AI market for AI/ML/DL includes software embedded inside chip, device, module, network infrastructure, subsystem, information processing and integrated application. AI may be used in or applied to one of many industries and play as a tool of assistance. AI may also can be a kind of product. And we expect more products are coming out in the global market based on the amount of investment of AI R\&D activities in US, China, Europe, Japan, and Taiwan. At last. AI can be as a service (AaaS). We can see more and more AI plays as services and products by itself. AI will become one of the most promising high technology industries.

\section{Toward an AI-enabled SDN-based 5G \&IoT Network}

This section we focus on the architecture, design, and challenge issues.

\subsection{Architecture of AI-Enabled 5G/IoT Network}

Figure 6 and 7 have shown the simplified architecture of SDN-based 5G/IoT: Figure 6 without AI and Figure 7 with AI/.ML. The intelligent agents can be applied to each layer of four-layer model in Figure 4. In this case, it applies to network infrastructure \& construction layer. Figure 7 illustrates AI/ML has been applied to information processing and integration application. Together with Figures 4, 5, 6, 7 and 8, the architecture of AI-enabled SDN-based 5G/IoT network is shown in Figure 9.

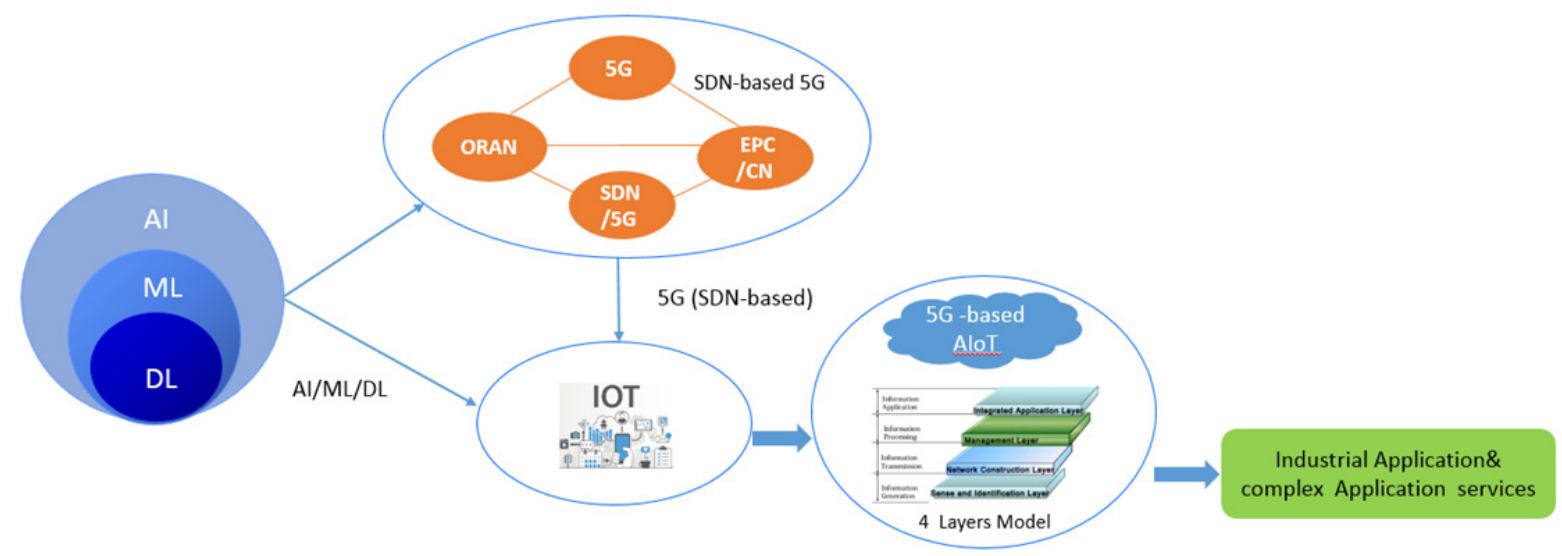

Figure 9. AI-enabled SDN-based 5G/IoT network 


\subsection{Design and Application of AI-enabled 5G/IoT Network}

The design of SDN-based AI-enabled 5G/IoT based on the architecture of AI-enabled 5G/IoT of Figure 9. To ensure the global level optimization, both $5 \mathrm{G}$ and IoT have to go through AI/ML/DL learning process.

For the applications of AI-enabled 5G/IoT Network, the fusion of AI, 5G, and IoT creates the new opportunities of smart agriculture or farms, smart healthcare, intelligent transportation, smart city. Figure 10 shows the process and applications created by the integration of 5G, AI, and IoT.

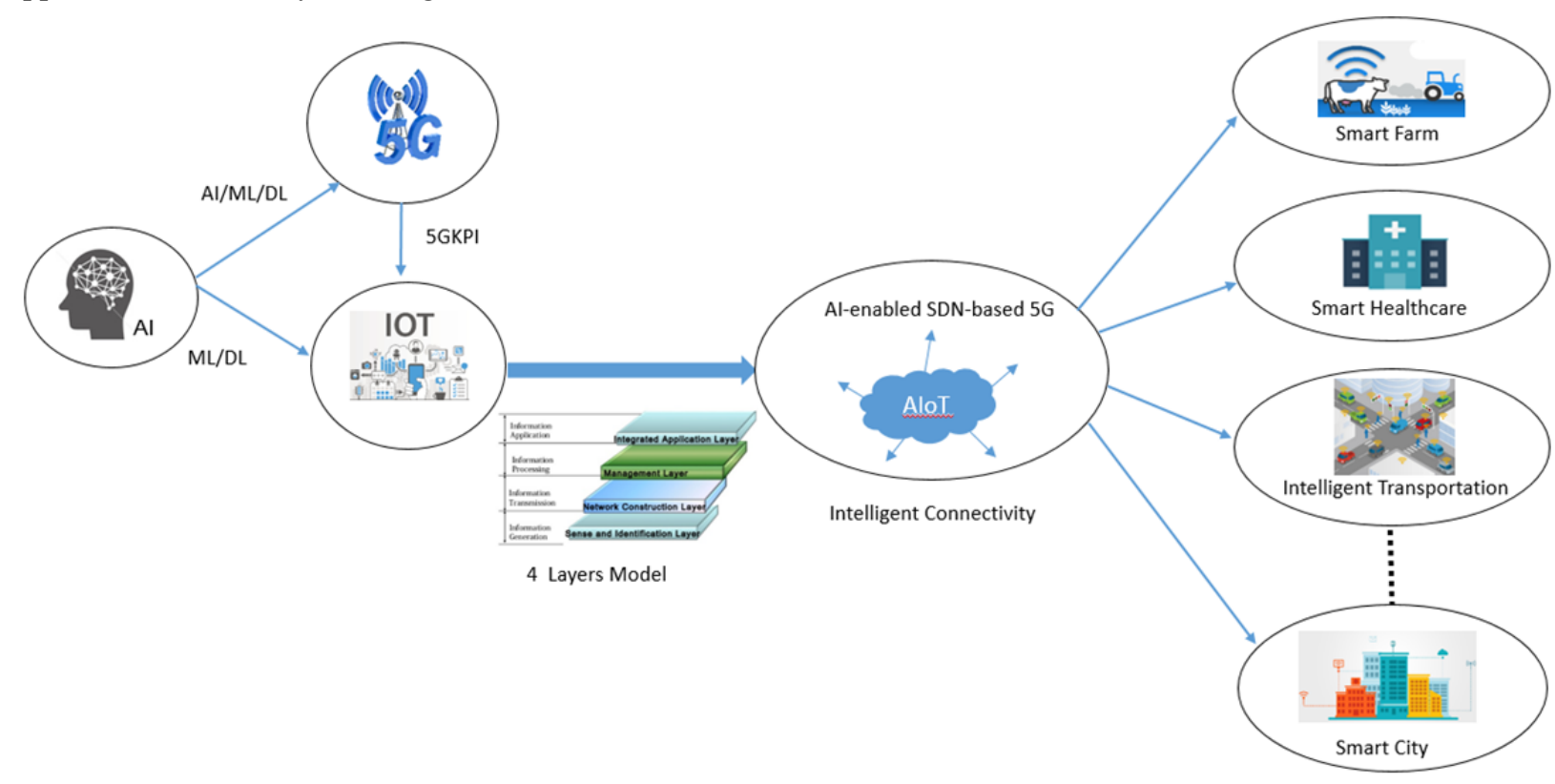

Figure10. Open Intelligent connectivity

\subsection{The Challenge in the Realization of AI-enabled 5G/IoT Network}

The successful event of Japan's Rakuren Mobile awarded a 5G license by using Open Networking (ON) architecture on April 11, 2019. But Rakuren has been delayed in the deployment phase expected to occur in June 2020. The challenges in the implementation is still remained:

1) Softwarization and virtualization (SDN/NFV) of open source5G availability (5G Americas whitepaper, 2019)

2) The software quality of open source code

3) The completeness of open source for $5 \mathrm{G}$ (Snow \& Parulkar, 2016)

4) More successful stories for field trials and network deployment needed

5) AI-enabled to identify assistant role or proactive role

\section{Future Work \& Conclusion}

This article addresses a variety of industries having been Alized by applying AI/ML/DL techniques to act as a tool or to show as an assistant to enhance the other industries. It is not clear what AI industry means. When they are more $\mathrm{AI} / \mathrm{ML} / \mathrm{DL}$ technology used to create or produces more differentiable and unique AI-based products and applications/services, then AI will have its own industry, AI Industry will be one of competitive technology industries.

\section{Acknowledgments}

This work was supported by the Ministry of Science and Technology (MOST) of Taiwan and National Chiao Tung University under grants: MOST 109-2221-E-009-081- , 108-2221-E-009-043-.

\section{References}

$5 G$ Americas whitepaper. (2019). The status of Open Source 5G. Retrieved from https://www.5gamericas.org/wp-content/uploads/2019/07/5G_Americas_White_Paper_The_Status_of_Ope n_Source_for_5G_Feb_2019.pdf

Akyildiz, I. F. (2017). Internet of Things: Trends, Directions, Opportunities, Challenges. BWN Lab, School of 
ECE, Georgia Institute of Technology, IFA'2017. Retrieved from http:/www.iotunisia.com/wp-content/uploads/2017/08/INTERNET-OF-THINGS-TRENDS_DIRECTIONS _OPPOTUNITIES_CHALLENGES-by-IFA.pdf

ATIS. (2018). Evolution to an Artificial Intelligence - Enabled Network. ATIS. Retrieved Sept. 2018, from access.atis.org

CNBC. (2019). Japan's Rakuten to roll out 5 G services by June 2020, CEO says. Retrieved August 3, 2019, from https://www.cnbc.com/2019/08/01/rakuten-to-roll-out-5g-services-by-june-2020-ceo-hiroshi-mikitani.html

Latah, M., \& Toker, L. (2018). Artificial intelligence enabled software-defined networking: a comprehensive overview. IET Networks, 8(2), 79-99.

Lin, B. S. (2019) Exploring The Next Generation IoT in The 5 G and AI Era. Keynote Speech, FU-JEN University, College of Science and Engineering, EE\&CS. Retrieved May 23, 2019, from https:/www.routledge.com/5G-Enabled-Internet-of-Things/Wu-Huang-Wang-Pan/p/book/9780367190101

Lin, B. S. P., Lin, F. J., \& Tung, L. P. (2016). The roles of 5G mobile broadband in the development of IoT, big data, cloud and SDN. Communications and Network, 8(1), 9.

Lin, B. S., Lin, Y. B., Tung, L. P., \& Lin, F. J. (2019). Chapter 4 -Exploring the Next Generation of the Internet of Things in the $5 \mathrm{G}$ Era. 5G-Enabled Internet of Things, 67. CRC Press.

Martinez-Julia, P., \& Skarmeta, A. F. (2014). Empowering the internet of things with software defined networking. White Paper, IoT6-FP7 European research project. Retrieved from https://www.iot6.eu/sites/default/files/imageblock/IoT6\%20-\%20SDN\%20-\%20IoT.pdf

Martinez-Julia, P., \& Skarmeta, A. F. (2014). Empowering the internet of things with software defined networking. White Paper, IoT6-FP7 European research project. Retrieved from https://www.routledge.com/5G-Enabled-Internet-of-Things/Wu-Huang-Wang-Pan/p/book/9780367190101

Nelson, P. (2020). How AI will be used to manage 5G networks. 7 April 2020 Network World. Retrieved from www.networkworld.com

Peterson, L., Al-Shabibi, A., Anshutz, T., Baker, S., Bavier, A., Das, S., ... \& Snow, W. (2016). Central office re-architected as a data center. IEEE Communications Magazine, 54(10), 96-101.

Telecomscom. (2019). All four operators are awarded $5 G$ licenses in Japan, with security conditions attached. Retrieved April 11, 2019, from https://telecoms.com/496883/all-four-operators-are-awarded-5g-licences-in-japan-with-security-conditionsattached/

Wilson, C. (2019). “SDN + AI: A Powerful Combo for Better Networks”: LightReading. Retrieved Sept. 11, 2019, from https:/www.lightreading.com/analytics/analytics-systems/sdn--ai-a-powerful-combo-for-better-networks/d/ $\mathrm{d}-\mathrm{id} / 725705$

Wu, Y., Huang, H., Wang, C. X., \& Pan, Y. (2019). 5G-Enabled Internet of Things. CRC Press. Retrieved from https:/www.routledge.com/5G-Enabled-Internet-of-Things/Wu-Huang-Wang-Pan/p/book/9780367190101

Yao, H., Mai, T., Xu, X., Zhang, P., Li, M., \& Liu, Y. (2018). NetworkAI: An intelligent network architecture for self-learning control strategies in software defined networks. IEEE Internet of Things Journal, 5(6), 4319-4327.

Zheng, Y., Li, Z., Zhang, J., Xu, S., \& Dhody, D. (n.d.). Network Artificial Intelligence (NAI). IETF 98 Chicago. Retrieved

from https://datatracker.ietf.org/meeting/98/materials/slides-98-opsawg-network-artificial-intelligence-01

\section{Copyrights}

Copyright for this article is retained by the author(s), with first publication rights granted to the journal.

This is an open-access article distributed under the terms and conditions of the Creative Commons Attribution license (http://creativecommons.org/licenses/by/4.0/). 